\title{
FDM Exploitation for Next Access and Data Networks
}

\author{
Alberto Gatto, Paola Parolari, and Pierpaolo Boffi, Member, IEEE \\ Politecnico di Milano, Dept. Electronics, Information and Bioengineering, \\ PoliCom Lab, Via G. Ponzio 34/5, 20133 Milan, Italy \\ Tel: +390223998923 e-mail: pierpaolo.boffi@polimi.it
}

\begin{abstract}
By means of the dynamic and flexible choice of subcarrier modulation and spacing, frequency division multiplexing is demonstrated to overcome the system bandwidth limits achieving high capacity, both for datacenter interconnections and for multiple access passive optical networks.
\end{abstract}

Keywords: optical communications, frequency division multiplexing, access network.

\section{INTRODUCTION}

To face the rapid increase of traffic demand in short reach fibre communication networks, such as in intra- and inter-datacentre connections, and in medium reach transport, as in case of passive optical networks (PONs), where high capacity, low cost and power efficiency are mandatory, the standard approach based on single optical carrier modulation does not seem to be the best choice. The exploitation of a multicarrier approach, still in the single optical carrier, combined with direct detection (DD) can be an advantageous solution supported by the availability of high-speed digital signal processing (DSP), enabling a suitable signal spectrum design.

Orthogonal frequency division multiplexing (OFDM) [1,2] and its directly-modulated version discrete multitone (DMT) modulation provide a high spectral efficiency thanks to the partial subcarrier overlapping. Moreover, bit loading allows to minimize the penalty due to signal-to-signal beating interference. However, OFDM and DMT show the disadvantage of a significant peak-to-average power ratio (PAPR), that can induce possible signal distortions [3].

On the other hand, frequency division multiplexing (FDM) [4] appears as an interesting alternative thanks to its intrinsic flexibility in terms of subcarriers allocation and modulation. In particular, when multiple access is considered, FDM can be particularly interesting because just one subcarrier or a subset of subcarriers can be devoted to individual users and applications services. With respect to OFDM and DMT, a limited number of subcarriers are available with the necessity of a suitable spectrum shaping to increase the spectral efficiency.

In this paper, thanks to suitable experimentation, we highlight the FDM capabilities in some different applications:

- high capacity data links, where in presence of uncompensated fibre with DD and direct modulation (DM) FDM allows to overcome the bandwidth limitations of the employed system devices;

- PONs, where we exploit FDM flexibility to maximize the transported capacity and the network reach and to optimize the simultaneous access of multiple users.

\section{HIGH CAPACITY DATA INTERCONNECTIONS}

Present high capacity data communications systems targeting up to few tens of kilometres are based on DD and DM since they are very sensitive to cost, footprint and power consumption. FDM allows to achieve high capacity, also in case of the employment of low-cost devices, such as directly-modulated vertical-cavity surfaceemitting lasers (VCSELs) [5,6] characterized by high frequency chirp. In particular, in our experimentation we take into account a low-cost directly-modulated long-wavelength VCSEL [7] with a limited 3-dB electro-optical (E/O) bandwidth of $5 \mathrm{GHz}$ and 8-MHz linewidth. The laser frequency chirp and the double-sideband spectrum due to the VCSEL DM induce a non-uniform electrical frequency response after the propagation over an uncompensated single-mode fibre (SMF) span constituting the data link. In Fig. 1(left), the experimented transfer function of the whole transmission system is shown for different values of the propagated reach (back-to-back, $10 \mathrm{~km}$ and $20 \mathrm{~km}$ ); frequency dips, induced by the cumulated CD, appear around $6 \mathrm{GHz}$ and $4 \mathrm{GHz}$ for $10-\mathrm{km}$ and $20-\mathrm{km}$ SSMF propagation, respectively.

The FDM exploitation allows to adopt a tailored subcarrier allocation and modulation strategy to match the bandwidth limitation of the VCSEL and the non-uniform frequency response of the system [8]. The experimented approach is based on the filling of the spectral dips by FDM subcarriers characterized by more robust modulation formats, such as binary PSK, while the transported capacity is maximized by exploiting 16QAM and QPSK modulation in the other spectral ranges.

Experimentally we check the behaviour in case of a FDM signal constituted by 10 adjacent subcarriers, equally spaced at $1 \mathrm{GHz}$ and each modulated at 1 Gbaud, with Nyquist pulse shaping (roll-off factor null). Just 250-MHz frequency gap is maintained at the origin to reduce the carrier $\times$ subcarriers interference due to direct detection, without any other inter-subcarrier guard bands. Simple equalization (9-taps FFE and 4-taps DFE) is applied at the receiver side after DD in order to compensate for the signal distortions due to the limited E/O bandwidth. 
Figure 1(right) shows the experimental results for 20-km SMF propagation: the received electrical spectrum of the 10 FDM subcarriers with their measured BER is reported, together with the correspondent received constellations. BER target below the $7 \%$ overhead FEC threshold of $3.8 \times 10^{-3}$ is considered. Thanks to the electrical equalization of the transmitted power spectra, no significant spread among the subcarriers behaviour is visible and all the 10 FDM subcarriers satisfy the BER threshold. The same procedure has been performed for optimizing the FDM subcarriers modulation and allocation in case of 100-m (a distance typical for intradatacenter communications) and after $10-\mathrm{km}$ SSMF propagation in presence of frequency fading occurring around $6 \mathrm{GHz}$ for this last distance.

The reported experimentation confirms that a tailored FDM subcarrier generation in terms of modulation format and allocation can overcome the VCSEL band limitation and to match the non-uniform frequency response of the system, demonstrating a whole transported capacity from a maximum value of $34 \mathrm{~Gb} / \mathrm{s}$ (for 100 $\mathrm{m}$ reach) to $25 \mathrm{~Gb} / \mathrm{s}$ over $20-\mathrm{km}$ uncompensated SSMF reach, even with a $5-\mathrm{GHz}$ band directly modulated VCSEL in case of DD.
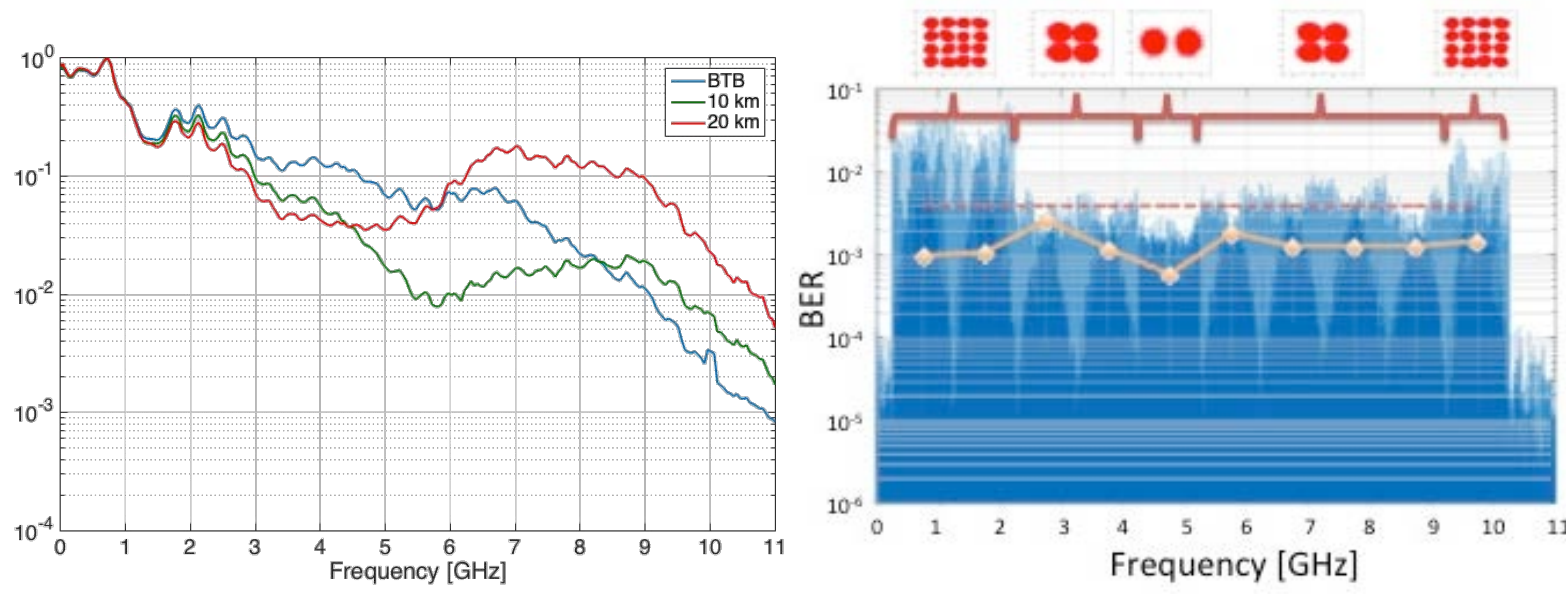

Figure 1: Left-Measured total transfer function due to uncompensated SFM propagation for different reaches, $B T B$ (blue line), $10 \mathrm{~km}$ (green line) and $20 \mathrm{~km}$ (red line); Right - 10-subcarriers FDM received electrical spectrum (blue) and subcarriers measured BER (orange) after 20-km SMF propagation (the BER threshold is the dashed red line). Above: the correspondent received constellations.

\section{PASSIVE OPTICAL NETWORKS}

The exploitation of FDM for the downstream (DS) and of FDM access (FDMA) for the upstream (US) for PONs applications is well known in literature for its offered intrinsic advantages [9]. By managing FDM subcarriers at the electrical level and considering an electrical local oscillator to detect each subcarrier in baseband, electronic simplification at the optical network unit (ONU) side is achieved with respect to the employment of standard time division multiplexing (TDM), that requires all ONUs transceivers to work at the full bit rate per wavelength. FDMA for the US allows the ONU to work only at its proper electrical spectrum slice, operating at the single-user rate. Thus, cost and power consumption reduction is guaranteed. Moreover, an increased spectral efficiency at least 3-4 times higher with respect to single carrier modulation employed with TDM can be achieved. Compared with OFDM, FDM doesn't require any synchronization for the subcarriers aggregation. Moreover, FDM flexibility allows to adapt the subcarriers to the channel characteristics and to the users requirements in a dynamic way. Such flexibility is a key enabling factor for future access networks.

In a PON a mandatory request is to reduce cost and complexity. In our experimentation, we analyse FDMAbased US, avoiding at the ONU the employment of a tuneable laser, but exploiting a low-cost device, such as a reflective semiconductor optical amplifier (RSOA) [10]. FDM adoption can face the limited-bandwidth of the RSOA, showing in the C-band a 3-dB bandwidth of $4 \mathrm{GHz}$. In Fig. 2 the experimented scheme for the US is shown, where the RSOA at the ONU is fed by an external continuous seed coming from the central office (CO), implementing the colourless solution thanks to the reflective approach.

A PON architecture compliant with the NG-PON2 standard layout can be realized, exploiting four WDM carriers to achieve a 4-fold increase in capacity compared to XG-PON and serving 64 users (16 user per WDM carrier sharing the same external seed) (Fig. 3). The approach adopted in our experimentation is based on the direct modulation of each RSOA to generate the FDM subcarriers of the US signal with the proper modulation and allocation tailored in order to match the bandwidth limitation of the RSOAs and of the other employed system devices. The external seeding is generated by a tunable laser with a $100-\mathrm{kHz}$ linewidth, split at the remote node $(\mathrm{RN})$ to reach the different RSOAs. To obtain the FDM carrier generation at each directlymodulated RSOA with Hermitian symmetric spectrum, we use Tektronix 50G/s arbitrary waveform generators with 14-GHz electrical bandwidth, each one driving the respective RSOA current. The DC bias current of both 
RSOAs is about $100 \mathrm{~mA}$. All the subcarriers generated by the different RSOAs at the same wavelength are combined at the RN to constitute the whole US FDM signal. At the CO coherent homodyne detection is implemented by means of the seed exploited as local oscillator. Single polarization detection is experimented either by exploiting an active polarization controller [11,12] or by using a $90^{\circ}$ polarization rotation due to the employment of a Faraday rotator at the input of a low-polarization dependent gain RSOA in order to achieve a retracing path for the polarization [10].

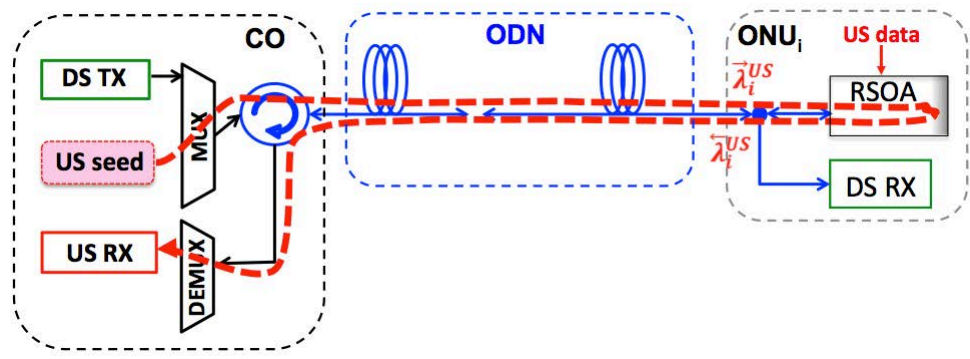

Figure 2. Reflective US scheme based on the exploitation of a RSOA at the ONU side fed by an external seed coming from the $C O$.

To reduce the optical beat noise between ONUs sharing the same wavelength seed it would be possible to exploit a balanced coherent detection [13] or a notch optical filter (NOF) combined to single-ended coherent detection. In our experimentation just a single-ended receiver is employed with a narrow fiber Bragg grating NOF with 1-GHz 3-dB bandwidth and about 15-dB notch depth inserted before coherent detection. The NOF is tuned to the seed wavelength, while no thermal control is applied, affecting the performance of the lowfrequency subcarriers. After O/E conversion, the detected FDM signal is acquired by a Tektronix oscilloscope DPO73304DX with $100 \mathrm{GS} / \mathrm{s}$ and 33-GHz electrical bandwidth to provide digital subcarriers demodulation and carrier phase recovery useful to check the system performance with respect to the subcarrier modulation. No digital equalization is present.

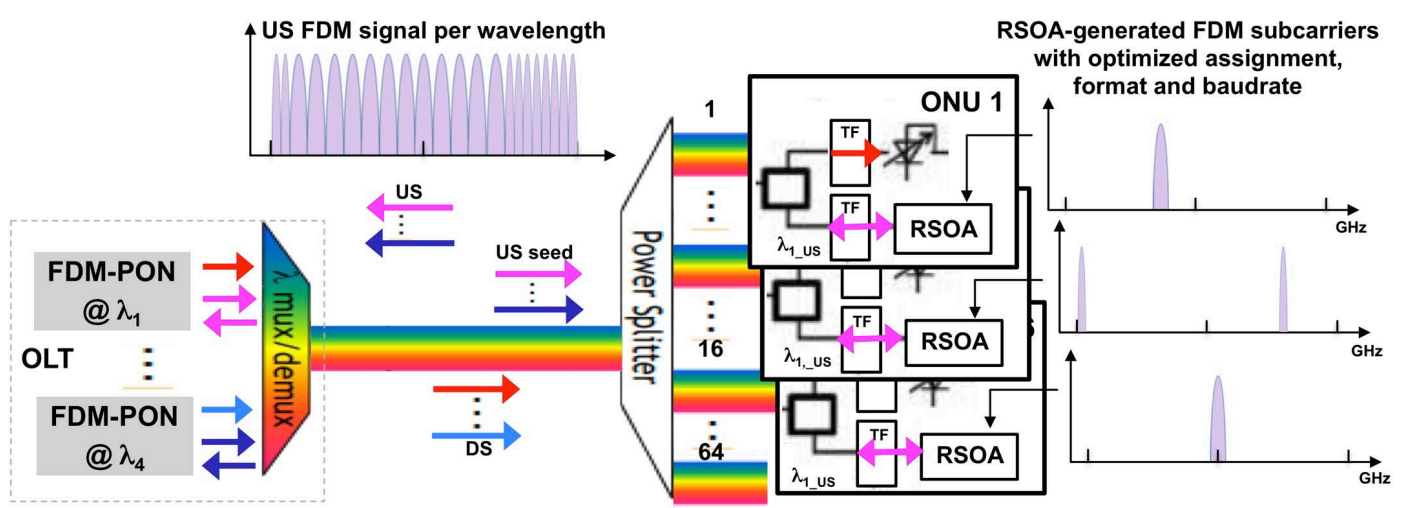

Figure 3. RSOA-based FDM PON scheme for US transmission compliant with NG-PON2 standard layout.

Figure 3 shows the case of 43-km uncompensated SMF propagation (corresponding to experimented 31-dB optical distribution network (ODN) losses). In groups of 16 ONUs, each RSOA is directly modulated at 500 Mbaud, in order to generate one among the 16 subcarriers constituting the US FDM signal per WDM carrier. No inter-subcarrier guard-band is inserted with an initial 1-GHz gap.

Taken 7\% overhead FEC BER threshold, the modulation of each subcarrier is optimized to maximize the whole US transported capacity. The central frequency subcarriers are 16QAM modulated, achieving 2 Gb/s data rate per ONU, while owing to the RSOA bandwidth limitation the higher frequency subcarriers are modulated using more robust QPSK modulation. QPSK subcarrier modulation is used also for the lower frequency range owing to the notch filter impact. In figure the performance in terms error vector magnitude (EVM) of the 16 FDM subcarriers is reported. The tailored choice of the modulation format of each subcarrier with respect to its proper spectrum allocation allows to satisfy the BER target for the subcarriers. In this way, over 43-km SMF, the whole capacity of $23 \mathrm{~Gb} / \mathrm{s}$ per wavelength is transported by 16 subcarriers in limited 9-GHz FDM signal spectrum, thus by means of 4 US WDM carriers an overall transported throughput of 92-Gb/s could be supported with about a tenfold increase in US capacity with respect to the NG-PON2 standard. 


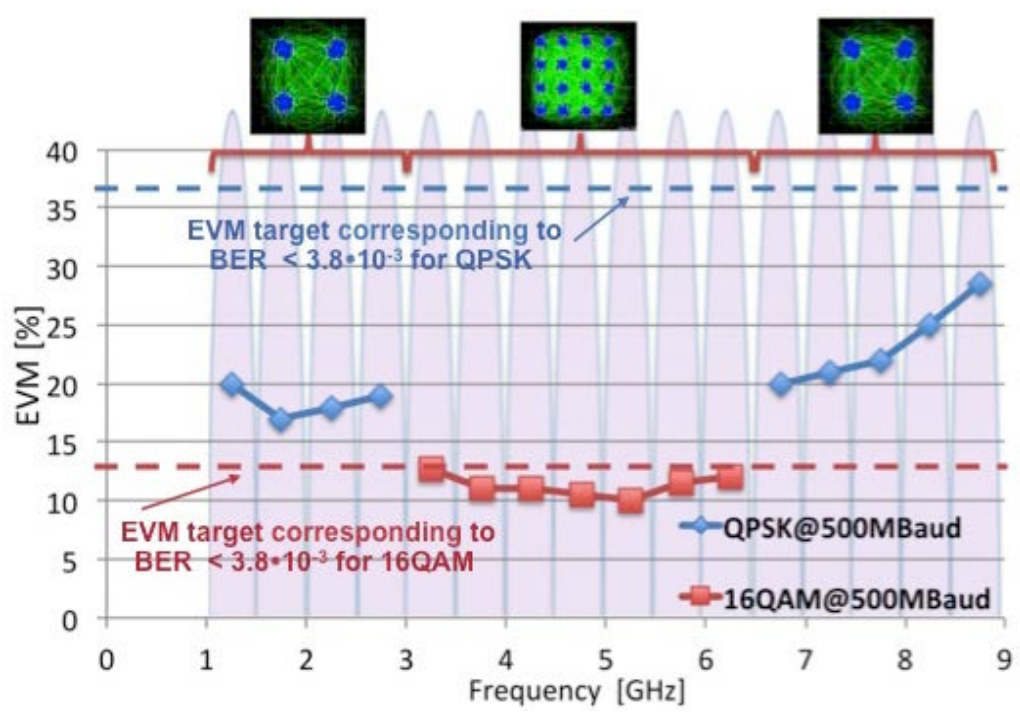

Figure 3. Measured EVM for each subcarrier of the experimented US FDM signal (500-MBaud modulation rate per each subcarrier) in case of 43-km SMF propagation. Dashed blue and red lines correspond to QPSK and $16 Q A M$ target, respectively $\left(3.8 \times 10^{-3} \mathrm{BER}\right)$.

\section{CONCLUSIONS}

In conclusion, we have shown recent progresses on the exploitation of FDM to achieve high transported capacity in few kilometres reach applications. We have shown FDM capabilities to face the bandwidth limitations of optical components, such as VCSELs and RSOAs, useful in the development of future low-cost and energyefficient systems. In particular, FDM intrinsic flexibility has been effectively used in case of multiple access.

\section{ACKNOWLEDGEMENTS}

The authors thank M. Brunero, P. Martelli, M. Martinelli of Politecnico di Milano for the support to the research activities. The authors thank also VERTILAS and TEKTRONIX for the support to experimentation.

\section{REFERENCES}

[1] J. Morosi, J. Hoxha, P. Martelli, P. Parolari, G. Cincotti, S. Shimizu, N. Wada, and P. Boffi, "25 Gbit/s per user coherent all-optical OFDM for Tbit/s-capable PONs," IEEE J. Opt. Commun. Netw., 8, 4, 190-195 (2016).

[2] P. Boffi et al., "Demonstration and performance investigation of hybrid OFDM systems for optical access network applications," IEEE Photon. J., 7, 1, 6987242 (2015).

[3] M. Sezer Erkilic et al., "Performance comparison of single-sideband direct detection Nyquist-subcarrier modulation and OFDM," J. Lightw. Technol. 33, 10, 2038-2046 (2015).

[4] A. Gatto, P. Parolari, and P. Boffi. "Frequency division multiplexing for very high capacity transmission in bandwidth-limited system," in Proc. OFC 2017, paper W1K.1 (2017).

[5] A. Gatto, A. Boletti, P. Boffi, and M. Martinelli, "Adjustable-chirp VCSEL-to-VCSEL injection locking for 10-Gb/s transmission at $1.55 \mu \mathrm{m}$," Opt. Express 17, 24, 21748-21753 (2009).

[6] P. Boffi A. Gatto, A. Boletti, P. Martelli, and M. Martinelli, "12.5Gbit/s VCSEL-based transmission over legacy MMFs by centre-launching technique," Electronics Letters 48, 20, 1289-1290 (2012).

[7] M. Ortsiefer et al., VCSELs - Springer Series in Optical Sciences 166 (Springer, 2013), Chap. 10.

[8] A. Gatto, D. Argenio, and P. Boffi, "Very high-capacity short-reach VCSEL systems exploiting multicarrier intensity modulation and direct detection," Opt. Express 24, 12769-12775 (2016).

[9] S. Straullu et al., "Optimization of reflective FDMA-PON architecture to achieve $32 \mathrm{~Gb} / \mathrm{s}$ per upstream wavelength over 31 dB ODN loss," J. Lightw. Technol. 33, 2, 474-480 (2015).

[10] A. Gatto et al., "RSOA-based FDM PON upstream with flexible multiple access capabilities in a NGPON2 compliant architecture," IEEE J. Opt. Commun. Netw. 8, 302-307 (2016).

[11] P. Martelli, P. Boffi, M. Ferrario, L. Marazzi, P. Parolari, S.M. Pietralunga, R. Siano, A. Righetti, and M. Martinelli, "Polarization stabilizer for polarization-division multiplexed optical systems," in Proc. $33^{\text {rd }}$ European Conference of Optical Communication (ECOC), Berlin - Germany (2007).

[12] P. Boffi et al., "Stable 100-Gb/s POLMUX-DQPSK transmission with automatic polarization stabilization," IEEE Photon. Technol. Lett. 21, 11, 745-747 (2009).

[13] Y.Y. Wona et al., "Suppression of optical beat interference-noise in orthogonal frequency division multiple access-passive optical network link using self-homodyne balanced detection," Opt. Fiber Technol. 20, 309313 (2014). 